\title{
Field-of-View Guiding Camera on the HISAKI (SPRINT-A) Satellite
}

\author{
A. Yamazaki · F. Tsuchiya · T. Sakanoi · K. Uemizu • K. Yoshioka • G. Murakami • \\ M. Kagitani · Y. Kasaba · I. Yoshikawa $\cdot$ N. Terada · T. Kimura · S. Sakai · \\ K. Nakaya $\cdot$ S. Fukuda $\cdot$ S. Sawai
}

Received: 25 April 2014 / Accepted: 27 September 2014 / Published online: 22 October 2014

(C) The Author(s) 2014. This article is published with open access at Springerlink.com

\begin{abstract}
HISAKI (SPRINT-A) satellite is an earth-orbiting Extreme UltraViolet (EUV) spectroscopic mission and launched on 14 Sep. 2013 by the launch vehicle Epsilon-1. Extreme ultraviolet spectroscope (EXCEED) onboard the satellite will investigate plasma dynamics in Jupiter's inner magnetosphere and atmospheric escape from Venus and Mars. EUV spectroscopy is useful to measure electron density and temperature and ion composition in plasma environment. EXCEED also has an advantage to measure spatial distribution of plasmas around the planets. To measure radial plasma distribution in the Jovian inner magnetosphere and plasma emissions from ionosphere, exosphere and tail separately (for Venus and Mars), the pointing accuracy of the spectroscope should be smaller than spatial structures of interest (20 arc-seconds). For satellites in the low earth orbit (LEO), the pointing displacement is generally caused by change of alignment between the satellite bus module and the telescope due to the changing thermal inputs from the Sun and Earth. The HISAKI satellite is designed to compensate the displacement by tracking the target with using a Field-Of-View (FOV) guiding camera. Initial checkout of the attitude control for the EXCEED observation shows that pointing accuracy kept within 2 arc-seconds in a case of "track mode" which is used for Jupiter observation. For observations of Mercury, Venus, Mars, and Saturn, the entire disk will be guided inside slit to observe plasma around the
\end{abstract}

A. Yamazaki $(\bowtie) \cdot$ K. Uemizu $\cdot$ K. Yoshioka · G. Murakami · T. Kimura · S. Sakai · K. Nakaya · S. Fukuda $\cdot$ S. Sawai

Institute of Space and Astronautical Science, Japan Aerospace Exploration Agency, Sagamihara, Japan e-mail: yamazaki@stp.isas.jaxa.jp

F. Tsuchiya $\cdot$ T. Sakanoi $\cdot$ M. Kagitani

Planetary Plasma and Atmospheric Research Center, Tohoku University, Sendai, Japan

K. Uemizu

The National Astronomical Observatory of Japan (NAOJ), Mitaka, Japan

I. Yoshikawa

Department of Earth and Planetary Science, The University of Tokyo, Tokyo, Japan

Y. Kasaba $\cdot$ N. Terada

Department of Geophysics, Tohoku University, Sendai, Japan 
planets. Since the FOV camera does not capture the disk in this case, the satellite uses a star tracker (STT) to hold the attitude ("hold mode"). Pointing accuracy during this mode has been $20-25$ arc-seconds. It has been confirmed that the attitude control works well as designed.

Keywords HISAKI satellite $\cdot$ Telescope pointing accuracy Extreme ultraviolet spectroscope $\cdot$ Solar system planet

\section{Introduction}

The planets of our solar system hold each unique environment owing to the pressure balance with the solar wind dynamic and magnetic pressures. The counteracting pressure in the side of planetary atmosphere or magnetosphere is plasma and/or magnetic pressures. The circumplanetary plasma distribution is controlled by the strength of the intrinsic magnetic field and the plasma abundance and temperature. The magnetic field strength and rotation rate of planet, especially, should be sufficient parameters to characterize each planetary plasma environment. Jupiter has the strongest intrinsic magnetic field and fastest rotation rate in our solar system, and Venus has the weakest field.

Primary source of plasma in the Jovian magnetosphere is Io plasma torus (IPT) where heavy ions such as sulfur and oxygen originated from the satellite Io distribute along its orbit at $5.9 R_{J}$ ( $R_{J}$ is Jovian radius) from Jupiter (Thomas et al. 2004). The plasma is slowly transported outward and accelerates to enforce the plasma flow rotating around the planet due to angular momentum transfer from the planet to the plasma via magnetosphereionosphere coupling current system (e.g., Hill 1979). As a result, the plasma convection in the magnetosphere is dominated by the rotation of the planet (Krupp et al. 2001; Cowley et al. 2003) and the size of the magnetosphere expands up to $100 \mathrm{R}_{\mathrm{J}}$ toward the subsolar direction due to the contribution of internal plasma pressure (Huddleston et al. 1998). While it has been thought that the influence of the solar wind on this rotation dominant magnetosphere is limited, several observational evidences which show significant effect of the solar wind on inner and middle magnetospheres exist (Reiner et al. 2000; Khurana 2001; Tao et al. 2005; Nozawa et al. 2006; Tsuchiya et al. 2011) and this issue is still open and regarded as one of outstanding questions of the Jovian magnetosphere (e.g., Krupp et al. 2004).

At circum-Venus, because of an absence of the intrinsic magnetic field, the solar wind dynamic pressure balances with the plasma pressure of Venus ionosphere. The direct interaction between the solar wind and the Venus ionosphere results into atmospheric escape to interplanetary space (e.g., Luhmann and Kozyra 1991; Lundin 2011). The many numerical simulation results show that the heavy ions such as oxygen, carbon, and nitrogen ions, can escape to interplanetary space from the planetary gravity in non-thermal escape processes (e.g., Tanaka and Murawski 1997; Terada et al. 2002). The large amount escape of heavy ions from the ionosphere and the atmosphere were clarified as observational results of several spacecraft such as the Pioneer Venus Orbiter (PVO) (Brace et al. 1982) and Venus Express (Barabash et al. 2007; Luhmann et al. 2008). However because the results are based on data obtained by the in-situ measurements, the total escaping rate from the whole planet of a macroscopic quantity is estimated by an assumption that atmospheric escape occurs uniformly. Also the escape rate could depend on the solar activity. To examine the depen- 
dence of the escape mechanisms on the solar activity leads to knowledge about the planetary atmospheric evolution from the early days of the solar system (Terada et al. 2009a).

The comparison between the solar wind dynamic and plasma pressures around these two planets contains substantial evidence for differences of each planetary plasma environment of our solar system. There are two types of plasma observation method in planetary atmospheres and magnetospheres, one is in-situ observation and the other is the remote sensing. The in-situ observation is useful for obtaining the microscopic physical quantity, and the remote sensing is suitable for measurement of the macroscopic quantity. The extreme ultraviolet (EUV) optical observation is appropriate for the global plasma environments. It is because the transition energy of the plasma in the planetary atmosphere and magnetosphere is ground state and because the transition energy is the same as the EUV photon energy. Only the remote sensing measurement can measure sources of plasma quantitatively and responses of the environment around planets to the solar wind at multi-planetary condition using the same instrument.

The HISAKI (SPRINT-A) Satellite (Sawai et al. 2014) carries the EUV imaging spectroscope instrument optimized for planetary observation (Yoshioka et al. 2013). It is the first small scientific satellite of the Japan Aerospace Exploration Agency (JAXA) using the standard bus system (Fukuda et al. 2008; Nakaya et al. 2011), and is aimed at achieving an ultraviolet spectroscope (EXCEED) mission for the planetary science on Jupiter's magnetosphere and the atmospheric escape from the Earth-type planets, Venus and Mars (Yoshikawa et al. 2014). It was launched on 14 September 2013 from the Uchinoura Space Center by the launch vehicle Epsilon-1 (Morita 2012), and was successfully inserted into the observational orbit of the low earth orbit with an apogee of $1157 \mathrm{~km}$, a perigee of $954 \mathrm{~km}$, and an inclination of 29.7 degrees. After an initial check out operation during about two months, scientific observations have started to obtain initial EUV spectrographic results of plasma environments around Jupiter and Venus (Yoshikawa et al. 2014).

Additional equipment of a Field-Of-View (FOV) guiding camera is installed on the mission module of the satellite to satisfy scientific requirements such as a pointing accuracy. The camera is associated with an attitude control system of the satellite, and guides the EUV instrument's field-of-view on the observed planet. The scientific requirement for spatial resolution is 20 arc-seconds, which is derived from the following scientific themes. In the observation of the Jupiter's magnetosphere, in order to clarify the radial structure of plasma temperature and density in the IPT, the spatial resolution higher than half the size of the IPT width viewed from the Earth, which is about 40 arc-seconds at the closest point, is one of most essential performances. As for observations around Venus and Mars, a separate detection of emissions from ionosphere, exosphere, and tail is necessary. Its condition is included in the requirement of the Jupiter's observation. However the pointing accuracy of 20 arcseconds by the observational condition exceeds a performance of a common satellite of a size similar with this small scientific satellite. According to the results of the prelaunch system analysis, it is predicted that the attitude pointing accuracy is about $+/-2$ arc-minutes in using only the attitude and orbital control system (AOCS) on the bus module of the satellite (Sakai et al. 2011). Therefore an install on the mission module of the additional equipment, the FOV camera, is adopted. The FOV camera image data of the observed planet transfer to a Mission Data Processor (MDP), and MDP analyzes the image to calculate the centroid of the image. The centroid data are used for the feedback control of the AOCS system to avoid blurring images due to thermal strain and thermal shock of the satellite body. The details of control algorithms using the FOV camera's centroid data at the attitude and orbital control system on the bus system of the satellite are described in another paper (Sawai et al. 2014). 


\section{Instrumentation Description}

\subsection{Design and Function}

The FOV camera, one of the focal plane assemblies, is a slit plate viewer at the visible wavelength, which is acquiring and guiding a planet image to the slit for the EUV spectrograph (Yoshioka et al. 2013). The optical layout is shown in Fig. 1.

Light emitted from the planet are gathered to the primary focal plane of the entrance mirror, which is an off-axis parabolic mirror with $200 \mathrm{~mm}$ of an effective diameter and $1600 \mathrm{~mm}$ of a focal length. The light on the field surrounding the slit on the focal plane is reflected off the slit plate, and is guided into the slit viewer. The FOV camera consists of a collimating lens, an aperture stop, camera lens, a filter, and a sensor module. All the optical components aligned coaxially in a cylindrical structure made of aluminum alloy applying black paint. Achromatic doublet lens are adopted as the collimating and camera lenses, whose focal lengths are 200 and $400 \mathrm{~mm}$ respectively to enlarge the image scale double. The diameter of the aperture stop is $21.5 \mathrm{~mm}$. Light goes through a narrow band-pass filter, which is centered at $553 \mathrm{~nm}$ with a 35-nm width (FWHM: full width of half maximum), to the detector array. The FOV camera weights $1.4 \mathrm{~kg}$ in total. A photograph of the installed FOV camera is shown in Fig. 2. The final image quality is also dominated by aberrations of the telescope. Detail of the telescope and the slit module is described in Yoshioka et al. (2013).

The sensor module was initially adopted for a 1- $\mu$ m infrared (IR1) camera (Iwagami et al. 2011) on AKATSUKI, Japanese Venus Climate Orbiter, which was launched in 2010 (Nakamura et al. 2011), and the communication interface part is upgraded to use Space-Wire links for HISAKI. The detector array is a Si-CSD (charge sweeping device)/CCD which has $1040 \times 1040$ pixels with a pixel size of $17-\mu \mathrm{m}$ square. It has 4 output channels and a single output channel is used for readout of a quadrant to speed up the update rate in nominal operating. The architecture of the CSD device is based on a technology of the PtSi infrared (IR) imaging sensor, which was originally developed for IR imaging (Ueno 1996).

The FOV camera uses a sub frame of a $256 \times 256$-pixel box in one quadrant to obtain one image data, and it corresponds to a $280 \times 280$ arc-second field, thus its angular resolution is $1.1 \mathrm{arc}-\mathrm{second} / \mathrm{pixel}$. Its view angle size is enough wide for AOCS of the satellite to guide the observed planet into field-of-view of the FOV camera. The three types of slit are prepared on the basis of the scientific requirement (Yoshioka et al. 2013). In order to reduce the complexity of the guide mode algorithm, the position of the slit center line is aligned with the center of the FOV camera image in a vertical direction.

The mission module including the telescope and the focal plane assemblies is designed to be stabilized at the temperature of $20^{\circ} \mathrm{C}$ by heater control system for minimizing dis-

Fig. 1 The layout of the mission equipment originated in Yoshioka et al. (2013)

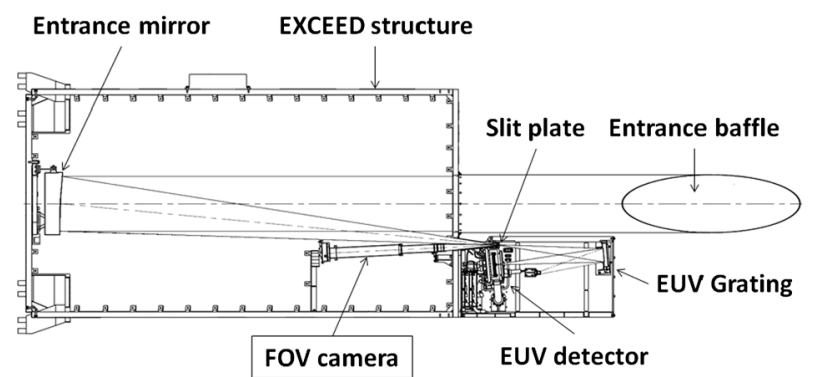


Fig. 2 A photograph of the FOV camera installed on the side panel of the satellite

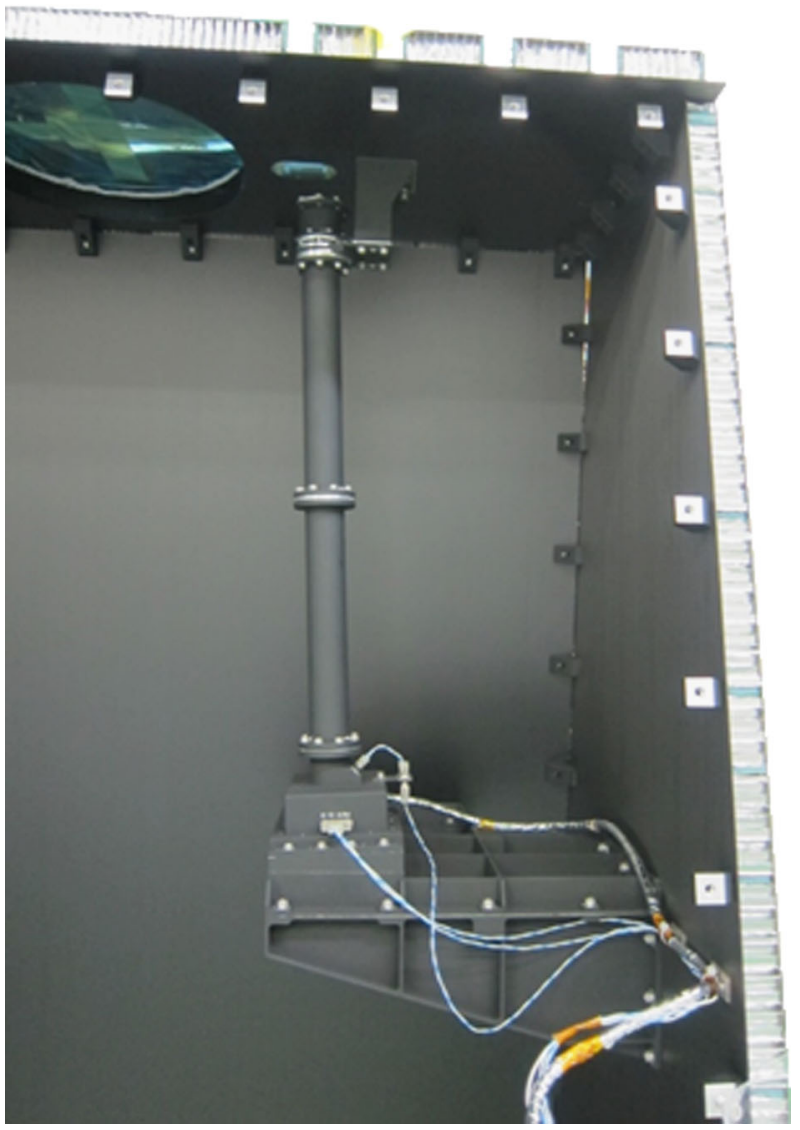

placement of focusing caused by the thermal expansion and mechanical deformations. The $\mathrm{Si}-\mathrm{SCD} / \mathrm{CCD}$ sensor is also kept in the $20{ }^{\circ} \mathrm{C}$ temperature, though the dark current is not negligible. Subtracting a dark frame can remove bright pixels in an image and adjust the threshold of brightness better against detection of dim targets.

The electronics of the FOV camera is installed in the box insulated thermally and mounted to a side panel of the mission module. It controls the Si-SCD/CCD sensor by the sequential operation command from MDP to capture signals of images, and transfers the 14-bit digital image data to MDP. The electronics consists of the power supply, the array drivers, the sequencer, the data acquisition hardware, the peripheral control, and Space-Wire links interface. The electronics including the insulated box weights $3.6 \mathrm{~kg}$.

The exposure time is controlled by the frame rate of the array operation, which is nominally 1 or 2 frames per second, thus the FOV camera does not have any mechanical shutter to make it robust in flight operation. The image of the observed target is updated every 3 seconds, and then a centroid position of bright target was calculated to recognize the fieldof-view direction of the FOV camera. The centroid data are sent to AOCS via MDP every 3 seconds to guide the observed planet within 5-arc-second (p-p) of the pointing accuracy of the satellite. The nominal exposure time is from 0.57 to 1.1 seconds at the observation such as Venus and Jupiter. The image of the FOV camera is downlinked in $2 \mathrm{~min} / \mathrm{image}$ in normal operating, which can be also used to confirm the health condition of the detector array and 


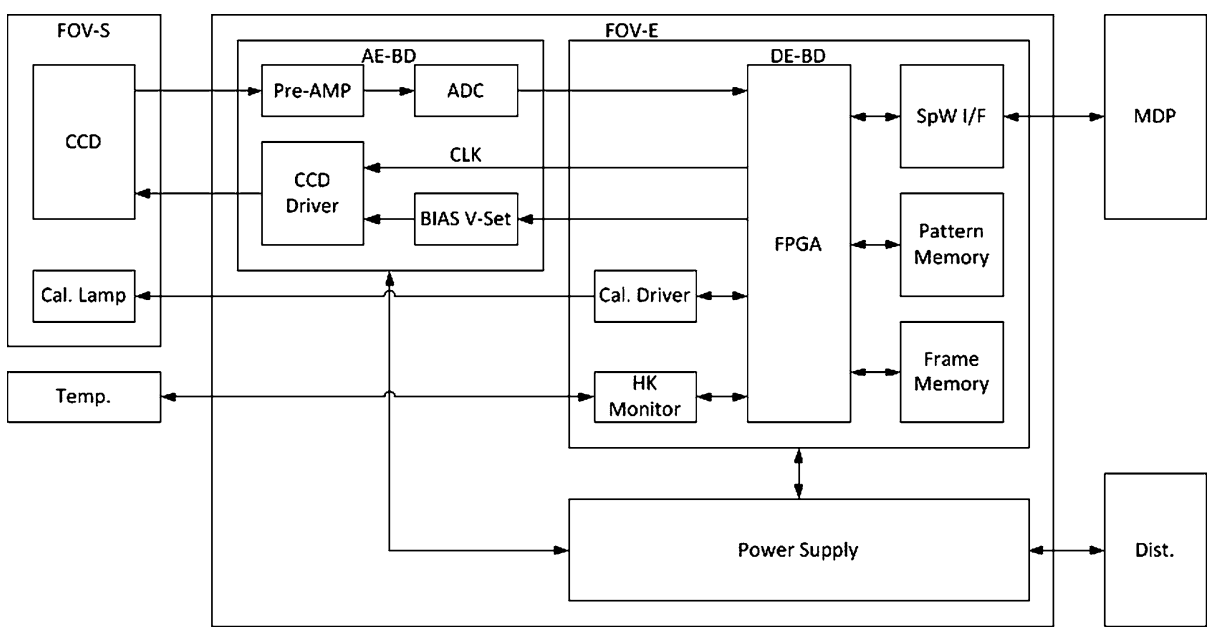

Fig. 3 A block diagram of the data stream for the optical and sensor part (FOV-S) and the electronics part (FOV-E) of the FOV camera

the pointing accuracy of the satellite attitude. A block diagram of the FOV camera is shown in Fig. 3.

\subsection{Performance and Test Results}

Both optical and electronics functional tests including sensitivity calibration are carried out using the optical and sensor part and the electronics part of the FOV camera flight model. The capability of the FOV camera was also evaluated by environmental tests, such as vibration and thermal vacuum tests, to be launched by the Epsilon rocket into the observational orbit. The integration test between the FOV camera and MDP is performed to evaluate the accuracy of centroid detection by measuring a disc-shaped bright object.

The two optical lenses of the collimating and camera lenses and the imaging SiSCD/CCD sensor were assembled with mechanical accuracy because the FOV camera does not require the determination of strict focal point due to an F-number as large as 16. The focal position in the vacuum conditions is adjusted by inserting appropriate shim plates.

Figure 4 shows an example of the dumbbell-shaped slit image taken by the FOV camera. As is shown a sharp slit image is obtained, and it is confirmed that the FOV camera takes images with sufficient quality for the purpose of centroid detection of an observed target.

The sensitivity of the FOV camera has been calibrated by an integrating sphere of National Institute of Polar Research in July 2012. Figure 5 shows a photograph of the calibration test. The reference intensity table of the integrating sphere has been calibrated and updated by manufacturer every year, the error in the reference intensity table is negligible. Furthermore this integrating sphere is optimized for rather faint light, such as aurora and airglow on the earth. The emission rate is from several $\mathrm{R}$ to hundreds $\mathrm{kR}$, where $\mathrm{R}$ is Rayleigh $\left(10^{6} / 4 / \pi\right.$ photons $\left./ \mathrm{cm}^{2} / \mathrm{s} / \mathrm{sr}\right)$. The intensity of planetary disk is so bright in the range of $\mathrm{MR} / \mathrm{nm}$ to $\mathrm{GR} / \mathrm{nm}$ that the sensitivity calibration data obtained using the integrating sphere may have ambiguity for such a bright object. It is believed, however, that the calibration data is still useful to estimate the counts of measuring planetary disk. From this calibration test, it is concluded that the sensitivity in the measurable range of the FOV camera is estimated to be $1.12 \times 10^{-5}[\mathrm{DN} / \mathrm{R} / \mathrm{pixel} / \mathrm{s}]$, where $1 \mathrm{DN}$ (Digital Number: A/D unit) corresponds to 
Fig. 4 An example of dumbbell-slit image taken by the FOV camera

Fig. 5 A sensitivity calibration test of the FOV camera
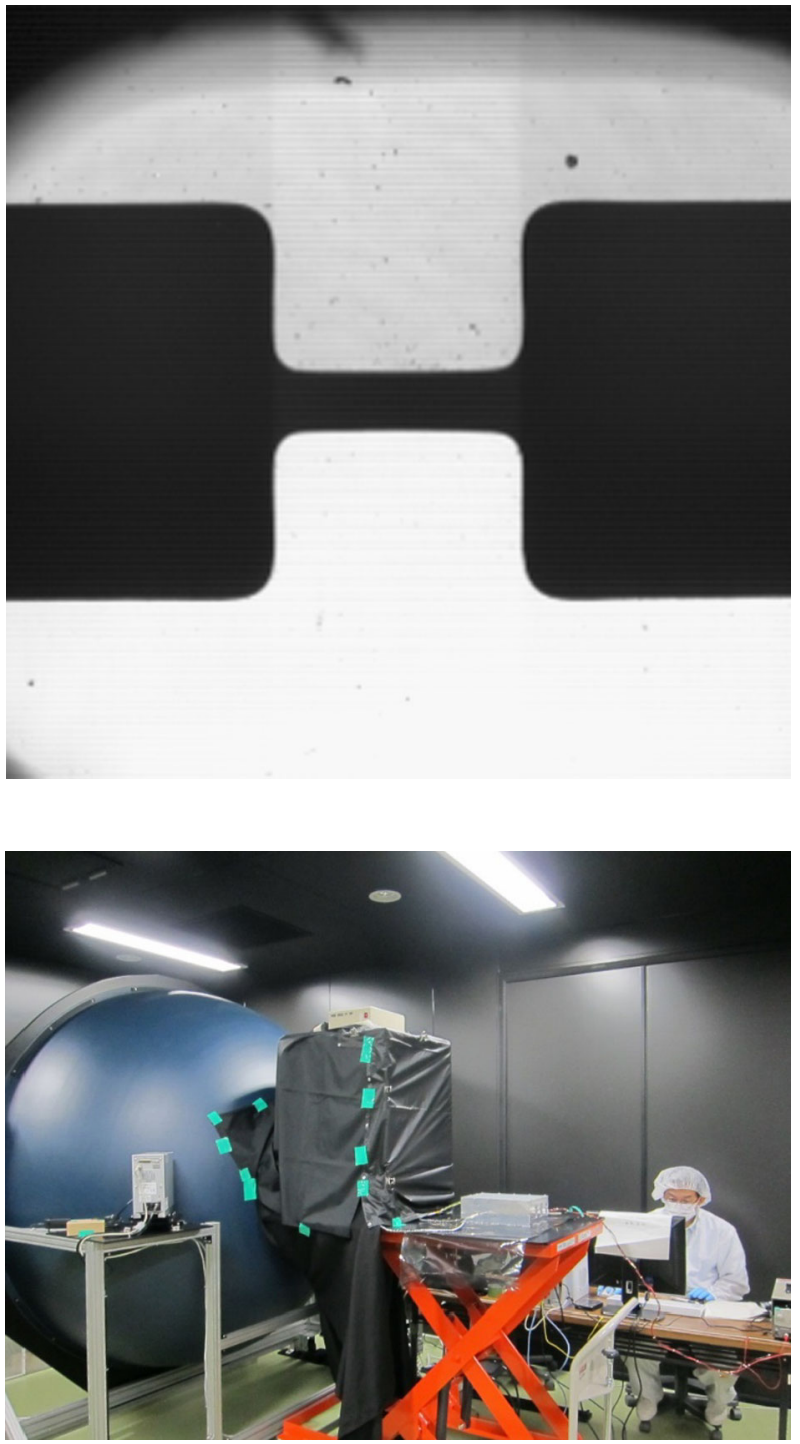

60 electrons. According to this sensitivity, as is summarized in Table 1, the count rate at the observation time is calculated to be $\sim 17000$ [DN/pixel/s] for Venus, 980 [DN/pixel/s] for Mars, and 260 [DN/pixel/s] for Jupiter. The result of calibration test is consistent with the count rate estimated with the intensity of planet and total throughput of optical instrument including the efficiency of detector, the bandwidth of filter, transmittance of lenses, and reflectance of mirror. And also the root-mean-square of the random read-out noise of image data taken by the FOV camera was measured to be 6 [DN/pixel] from these tests. Subtracting a dark frame, therefore, achieves the signal-to-noise ratio over 40, when Jupiter is acquired with an exposure time of 1 second. From the calibration test, it is confirmed that the FOV camera satisfies both the sensitivity for measuring planets with sufficient signal-to-noise ratio and the dynamic range of detector and $\mathrm{A} / \mathrm{D}$ unit. 
Table 1 Summary of estimated counts for Venus, Mars, and Jupiter. The emission intensity is estimated by the solar irradiance at each planet position on the assumption of the uniform reflection with the visual geometric albedo. The A/D unit is 60 electrons/DN. Calibration results are obtained with the target intensity and the sensitivity based on the pre-launch calibration results. Note that the estimated DN is applicable when the line-of sight is almost normal to incident solar flux, and significant reduction of DN should occur in the case of the inner planet Venus due to a large reflection angle of solar flux

\begin{tabular}{lrrr}
\hline Target & Venus & Mars & Jupiter \\
\hline Intensity at $500 \mathrm{~nm}[\mathrm{MR} / \mathrm{nm}]$ & 1900 & 110 & 29 \\
Calibration results $[\mathrm{DN} / \mathrm{pixel} / \mathrm{s}]$ & 17000 & 980 & 260 \\
\hline
\end{tabular}

After the successful launch of HISAKI, the FOV camera measured planets and obtained $14300[\mathrm{DN} / \mathrm{pixel} / \mathrm{s}]$ for Venus, 1170 [DN/pixel/s] for Mars, and 430 [DN/pixel/s] for Jupiter, where the value of DN/pixel/s is a peak count rate on each planetary disk. Jupiter and Mars were observed on 1 January and 6 April in 2014, respectively, and phase angles of both planets were near 0 degree. Venus was observed on 20 November in 2013, and the phase angle was 103 degrees. On Jupiter and Mars observations, the measured DN is slightly larger than those estimated from the pre-launch calibration test shown in Table 1. This discrepancy might be because the count rates estimated with the pre-launch calibration results were based on the assumption of uniform reflection on planetary surface. Although it is necessary to apply a reflectance curve against incident solar flux for the accurate estimation of planetary disk intensity, the reflectance is expected to be maximized at the normal incident solar flux, and decreases with increasing the angle between incident solar flux and the observational line-of-sight. This interpretation is consistent with the fact that the measured DN on Venus is smaller than that estimated from the pre-launch calibration results, because Venus is an inner planet and the phase angle between the line-of-sight and incident solar flux is large.

The thermal vacuum and the vibration tests for the FOV camera was carried out during the period from June to August 2012. The thermal vacuum test was performed to confirm the performance and function under vacuum at temperatures of the range from $-30{ }^{\circ} \mathrm{C}$ to $+60{ }^{\circ} \mathrm{C}$. The FOV camera passed all of the thermal vacuum and vibration tests. It was confirmed that there was no image quality shift, namely, the change in the focal point of the detector after the thermal vacuum and vibration tests.

The integration test between the FOV camera and MDP was carried out to evaluate the accuracy of centroid determination of target object in October 2012. The FOV camera obtained images of a pinhole with a diameter of $0.3 \mathrm{~mm}$ (35.3 pixels on the FOV camera image) as a disc-shaped target. MDP generated binary images of the pinhole by using an appropriate threshold level, and then calculated the centroid position of the images with onboard software. By comparing the manually estimated centroid position with one calculated by MDP, the consistency of the two centroid positions is confirmed. In addition, the immobilized pinhole images were acquired continuously for one hour to estimate the pointing accuracy and error in the centroid determination as an effect of only the FOV camera. From these tests, it is concluded that the centroid is determined with accuracy better than \pm 0.3 pixel, which corresponds to \pm 0.3 arc-seconds. The accuracy is sufficiently small and satisfies the requirement of centroid determination.

The FOV camera was installed on the satellite side panel in December 2012, as shown in Fig. 2. After the installation, the function test of the FOV camera was performed during the period from January to March 2013 to confirm the normal performance in atmospheric and vacuum conditions. As a result, there is no change in the image quality and the slit position 
after the installation on the satellite, and it is concluded that the specification of the FOV camera satisfies all of the requirements for guiding planetary targets.

\section{Image Processing}

\subsection{Overview of Target Finding and Pointing Correction}

A schematic picture in Fig. 6 shows the field-of-views of the slit and the FOV camera and the relative positions of Jupiter. Labels (1) to (3) in the figure show the sequence for capturing an observation target in the slit position. First of all, the satellite bus system locates the observation target to capture the bright reference target (planet disk) in the FOV camera (label (1) in Fig. 6(a)). MDP calculates the centroid of the target on the FOV camera image (star marks on the disk of labels (1)). When the planet disk overlaps with the edge of the field-of-view, the bus system carries out an attitude maneuver until the FOV camera captures the complete disk image. When the target disk falls completely in the slit, the maneuver is carried out in the direction perpendicular to the slit until the FOV camera captures the disk. After finding the target position, the bus system maneuvers the attitude so as to move the centroid of the target to the uplinked position (label (2)). The bus system controls the attitude to keep the centroid position of the target in the FOV camera (star mark in the disk of label (3)) with an accuracy of \pm 5 arc-seconds. This pointing correction algorithm is applied to correct slow changes in the pointing direction which is mainly caused by thermal strain and shock with changing thermal inputs from the Sun and Earth to the satellite. The vibrations which come from reaction wheels installed in the bus system are negligible for a factor that causes the pointing accuracy error of the HISAKI satellite because of its characteristics of a high frequency and randomness.

\subsection{Image Processing with Onboard Software}

Acquisition and processing of the FOV camera image and calculation of centroid position of the reference target are carried out with onboard software installed on MDP every 3 seconds.

Fig. 6 Schematic pictures showing the field-of-views of the dumbbell-shaped slit (white area) and the FOV camera (gray areas) and the relative positions of the reference target (Jovian disk). Labels (1) to (3) in the upper and lower panels show the sequence for capturing an observation target (the Jovian polar aurora and IPT) into the slit

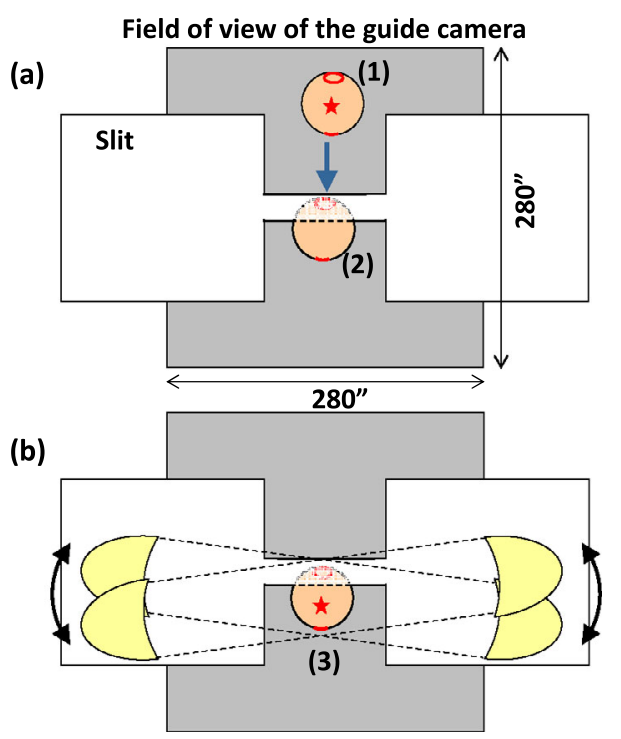




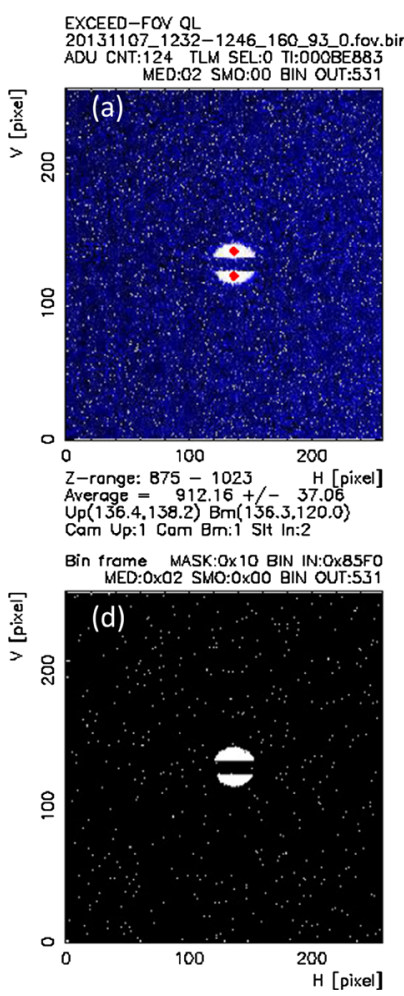

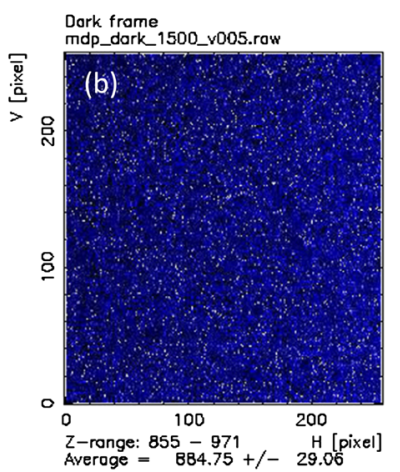

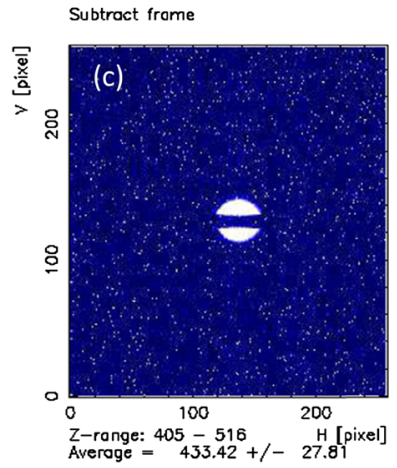

Bin frame (filtered)

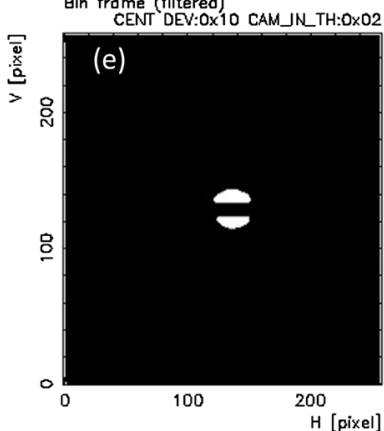

Fig. 7 An example of the image processing on MDP in a case of Jupiter observation with the $10^{\prime \prime}$ slit. The image was taken on Nov. 7, 2013 by the FOV camera

Fig. 8 Examples of count profile of the guide image along $H=128$ [pixel] column of Fig. 7(a)-(c)

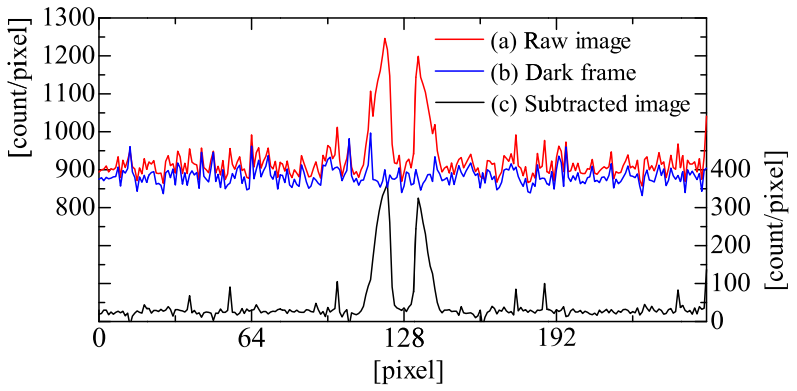

Procedure of the image processing is as follows; (1) Dark frame subtraction, (2) Smoothing to improve sensitivity (optional), (3) Binarization of the image, and (4) Noise filtering. Figure 7 shows an example of the image processing on MDP in a case of Jupiter observation with the $10^{\prime \prime}$ slit. The raw image shown in Fig. 7(a) contains the solar light reflected on Jupiter as well as biases associated with dark current of the image sensor, the fixed pattern noise which is associated with the read-out electric circuit, and hot pixels. To remove the fixed pattern noise and the permanent hot pixels, a dark frame shown in Fig. 7(b) is subtracted from the raw image. The dark frame is obtained by compositing several tens of images of dark sky and uploaded to the EEPROM on MDP. Figure 8(a)-(c) show count 
profiles of the guide image of Fig. 7(a)-(c), respectively, along $H=128$ [pixel] column. Signal-to-noise ratio of the image is significantly improved by subtracting the fixed pattern noise. To improve the sensitivity further, smoothing of the image is possible (this function will be used for Saturn, the darkest planet among the primary observation targets). After subtracting the dark frame, the image (Fig. 7(c)) is binarized (Fig. 7(d)) and centroid of bright part of the planet disk is calculated. Three kinds of computation method to determine the threshold level for the image binarization are installed in the software; (1) percentile method, one of well-used methods for image binarization, (2) direct setting of a threshold level by the command to MDP, and (3) average $+N$-sigma of the count in certain area of the FOV camera image. The method (3) is usually used for all observation targets. The average $(\bar{C})$ and standard deviation $(\sigma)$ of the count in $16 \times 16$ pixel areas at four corners of the image are calculated and the minimum among four values of $\bar{C}+N \sigma$ is selected as the threshold value. The value of $N$ (5 by default), area size $(16 \times 16$ pixels $)$, and offset from the corner ( 0 pixel) are set by the commands to MDP. In Fig. 7(d), a lot of isolated pixels whose values exceed $\bar{C}+N \sigma$ still remain on the image (white dots). To remove them, a median filter whose default kernel size is $3 \times 3$ pixels is applied (Fig. 7(e)). Finally, centroid of the reference target on the FOV camera $(\bar{H}, \bar{V})$ are calculated separately at upper and lower parts of the FOV camera with following equations;

$$
\bar{H}=\frac{1}{M N} \sum_{i}^{M} \sum_{j}^{N} C_{i j} H_{i}, \quad \bar{V}=\frac{1}{M N} \sum_{i}^{M} \sum_{j}^{N} C_{i j} V_{j}
$$

where $H_{i}$ and $V_{j}$ are pixel number of horizontal $(H)$ and vertical $(V)$ axes, respectively, $C_{i j}$ is value in the binarized image at $\left(H_{i}, V_{j}\right)$ pixel, $i=0$ to 255 , and $j=0$ to 127 and 128 to 255 for lower and upper parts of the image, respectively. Two red marks on Fig. 7 (a) are the centroid position calculated by MDP.

In the flight environment, high energy particles could cause bright spots on the FOV camera image near the South Atlantic Anomaly (SAA). These spots appear sporadically and the size of spots could be comparable to the apparent size of EUV stars and small planets such as Mercury. It is difficult to remove them by using the spatial median filter described above. To get rid of the sporadic spots, latest three successive binarized images are stored in the memory of MDP and median of the three images is taken for the value of each pixel.

\subsection{In-Flight Evaluation of Pointing Accuracy}

The HISAKI satellite has two kinds of attitude control mode for the planet observation: (1) Planet Tracking Control (PTC) mode and (2) in-Slit HOld (SHO) mode. In the PTC mode, the telescope pointing direction is controlled by using the centroid information. In the SHO mode, the satellite holds the attitude with star tracker (STT). The choice of the mode depends on the observation method of each planet. In the case of Jupiter observed by using the $10^{\prime \prime}$ slit, the slit is set to be parallel to IPT to measure radial spatial distribution of plasmas along the center of IPT (Fig. 9(a)). For observations with the 140" slit, it is kept to be parallel to the rotational equator and either northern or southern polar region is guided on the narrow part of the slit (Fig. 9(c)). Part of the Jupiter's disk is captured by the FOV camera as the diameter of the disk is larger than the slit widths and it is possible to track the planet by using the PTC mode. Other planets are nominally observed with the $60^{\prime \prime}$ slit to measure whole emissions around the planetary atmosphere and ionosphere (Fig. 9(b)). Because the bright disk falls into the slit and MDP cannot provide the attitude control system with the centroid information, the satellite holds the attitude by the SHO mode. In this case, 
Fig. 9 Schematic illustration of observations of IPT with $10^{\prime \prime}$ slit (a), IPT and Jupiter's northern aurora with $140^{\prime \prime}$ slit (dumbbell-shaped slit) (c), and Venus with $60^{\prime \prime}$ slit (b). Image of Jupiter and IPT are observed by Hubble space telescope (Clarke et al. 1996) and the Cassini spacecraft (Steffl et al. 2004). Image of Venus is based on the numerical simulation on the atmospheric escape (Terada et al. 2009b)
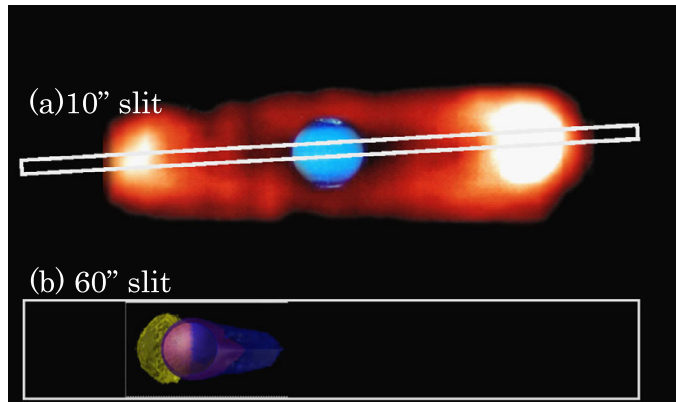

(c) $140 "$ slit

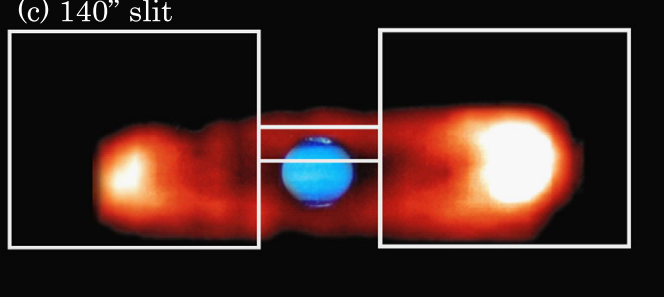

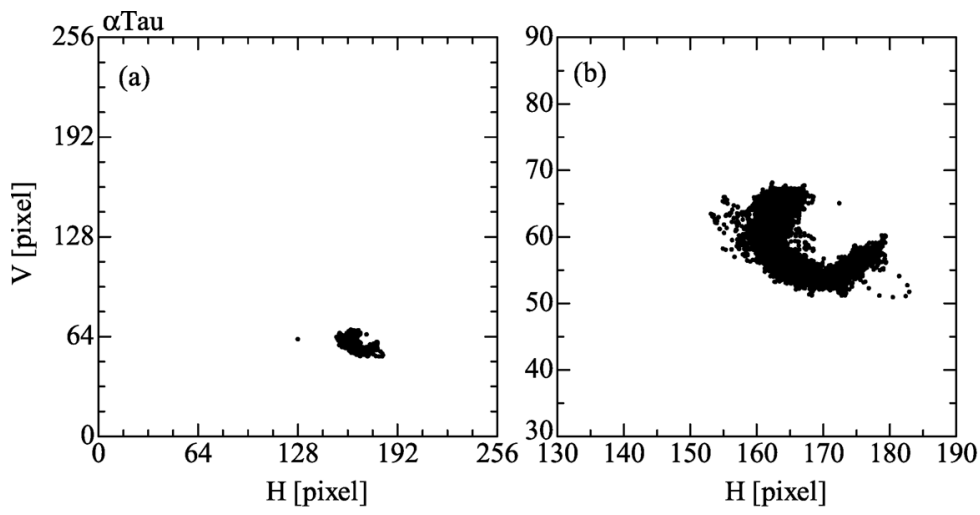

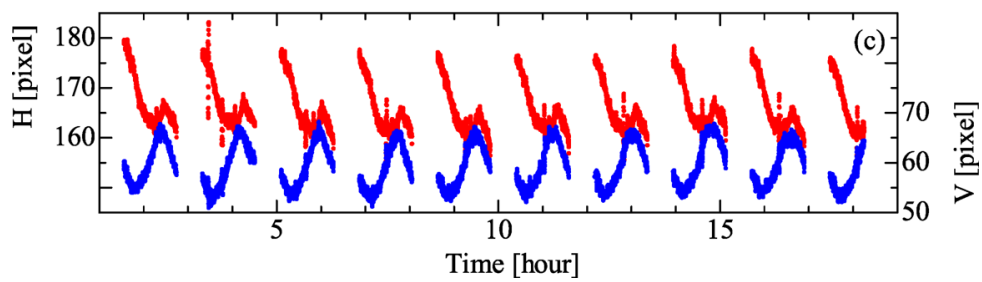

Fig. 10 (a) Centroid positions of $\alpha$ Tau taken by the FOV camera on Oct. 31, 2013. (b) The same as panel (a), but the $H$ and $V$ axes are expanded. (c) Time variation in centroid values ( $H:$ red and $V:$ blue)

displacement of the planet disk position could be caused by change of alignment between the satellite bus and the telescope.

Figure 10 shows variation in centroid positions during the SHO mode. The bright reference target is usually guided inside the slit and one cannot evaluate the actual position of the target with the FOV camera. To evaluate the pointing accuracy in the SHO mode, $\alpha$ Tau was captured on the FOV camera and the centroid position was monitored for one day. 

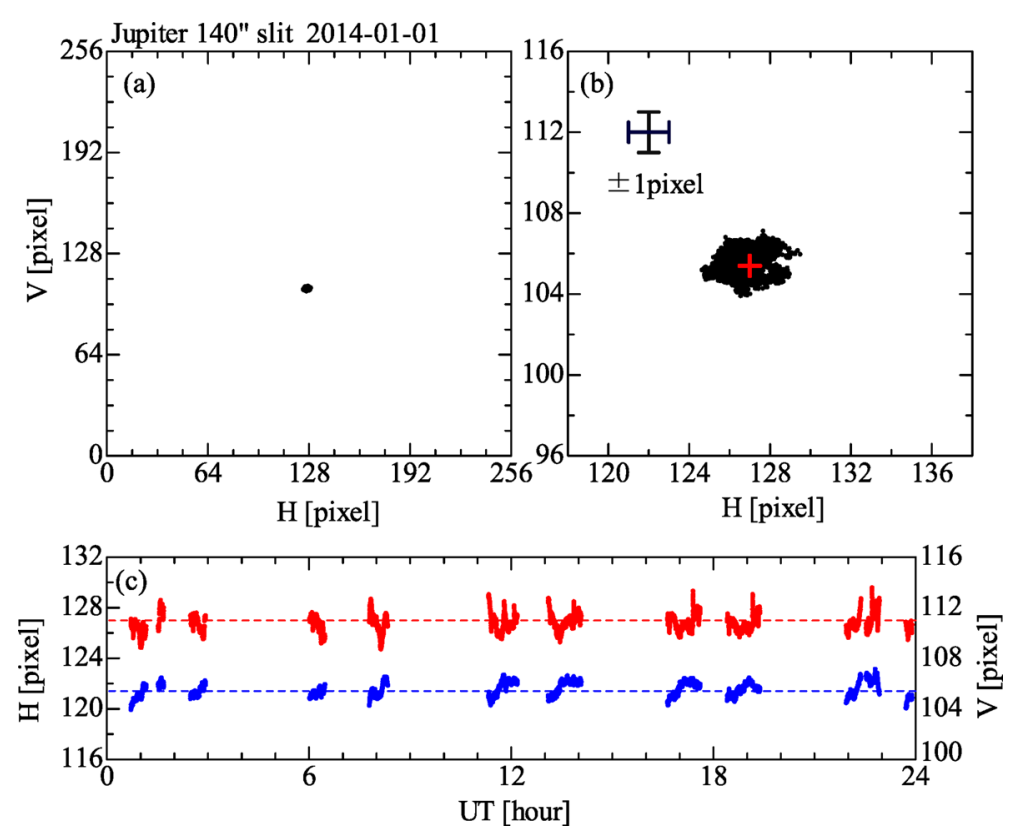

Fig. 11 (a) Centroid positions of Jupiter's disk taken by the FOV camera on Jan. 1, 2014. Only the data when high-voltage power supply for the EUV spectrograph is on are shown. (b) The same as panel (a), but the $H$ and $V$ axes are expanded. A red cross shows a target centroid position fed back for the attitude control. (c) Time variation in centroid position ( $H:$ red and $V:$ blue). Dashed lines show the target value for the attitude control. Data set are the same as panels (a) and (b)

Though MDP identified the centroid position and sent it to the attitude control system every 3 seconds, the attitude control system did not use it and kept the attitude with only STT. As shown in the panel (c), the position of the centroid showed periodic variation synchronized with the satellite orbital period of 106 minutes. This is caused by the change of alignment between the satellite bus and the telescope due to the periodic change of thermal inputs from the Sun and Earth to the satellite. The figure shows that the resultant pointing accuracy in the SHO mode was about 20-25 arc-seconds (the plate scale of the FOV camera is $1.1 \mathrm{arc}-\mathrm{second} / \mathrm{pixel})$.

Figure 11 shows variation in centroid positions of Jupiter on Jan. 1, 2014. During this period, as the satellite observed Jupiter with the $140^{\prime \prime}$ slit and only the northern polar region was guided into the slit, the centroid position was available only in the lower part of the FOV camera (Fig. 11(a)). The PTC mode was used for attitude control and the uplinked target position was $(H, V)=(127.0,105.4)$ (a red cross in the panel (b) and dashed lines in panel (c)). The panel (c) shows that the centroid position kept the target one with accuracy of a few arc-seconds and the displacement due to the change of the alignment was compensated. The resultant pointing accuracy was about a few arc-seconds, which is smaller than the spatial resolution of the EUV spectrograph itself (10 arc-seconds by pre-launch experiment; Yoshioka et al. 2013) and post-launch evaluation by using EUV stars in the SHO mode of 17 arc-seconds (Yoshikawa et al. 2014). Better spatial resolution is expected with the PTC mode and it will be evaluated in the near future.

Figure 12 shows frequency distributions of centroid values during the observation of Jupiter. Data set used for the analysis is the same as that shown in Fig. 11. From these distributions, the standard deviations from the uplinked position are calculated to be 0.79 and 
Fig. 12 Deviation of the centroid position of Jupiter from the uplinked position

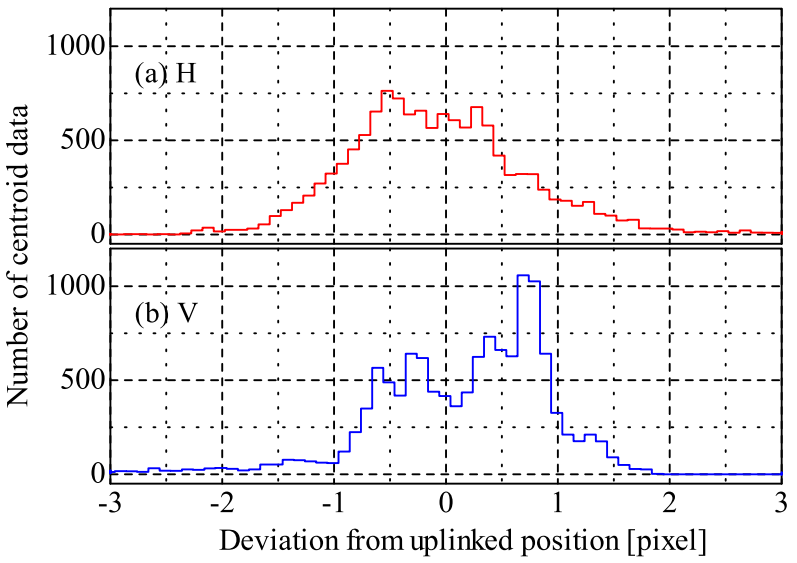

0.73 pixel for $H$ and $V$ directions, respectively. These values correspond to the Gaussian distribution of 1.85 and 1.71 pixels (FWHM), thus, to be 2.0 and 1.9 arc-seconds, respectively.

The centroid position derived onboard is downlinked to the ground and used to confirm the pointing accuracy and to further correct the position of EUV photons on the detector.

\section{Summary}

The first small scientific satellite of HISAKI (SPRINT-A) was launched by the Epsilon-1 rocket vehicle on 14 Sep. 2013, and was inserted into the low earth orbit. EXCEED on the satellite has successfully started the EUV observation after the initial check operation to gather continuous data of Jupiter's magnetospheric plasma distribution and the Venus atmospheric escape. The observation requires the advanced pointing accuracy of 20 arc-seconds for the small satellite. Therefore the additional equipment, the FOV camera, is installed on the mission module, and the centroid data are used for the feedback control of the AOCS system on the bus module of the satellite. The prelaunch calibration results confirm that the design, function, and performance of the FOV camera are suitable for the observation of planets such as Venus, Mars, and Jupiter. The flight data also show that the attitude pointing accuracy is 2.0 arc-seconds, which is smaller than the spatial resolution of the EUV spectrograph itself (about 10 arc-seconds by pre-launch experiment results and 17 arc-seconds by post-launch evaluation). The attitude pointing system incorporating the AOCS equipment on the bus module and the FOV camera on the mission module has successfully operated to achieve the pointing accuracy from the scientific requirement.

Acknowledgements The authors are grateful to Associate Professor Y. Ogawa, and Assistant Professor M.K. Ejiri of the National Institute of Polar Research for their fruitful collaboration about the pre-launch calibration test of the FOV camera. The authors also thank to all the members involved in the SPRINT-A project for their prodigious efforts to achieve the success of the scientific observations.

Open Access This article is distributed under the terms of the Creative Commons Attribution License which permits any use, distribution, and reproduction in any medium, provided the original author(s) and the source are credited. 


\section{References}

S. Barabash, A. Fedorov, J.J. Sauvaud, R. Lundin, C.T. Russell, Y. Futaana, T.L. Zhang, H. Andersson, K. Brinkfeldt, A. Grigoriev, M. Holmström, M. Yamauchi, K. Asamura, W. Baumjohann, H. Lammer, A.J. Coates, D.O. Kataria, D.R. Linder, C.C. Curtis, K.C. Hsieh, B.R. Sandel, M. Grande, H. Gunell, H.E.J. Koskinen, E. Kallio, P. Riihelä, T. Säles, W. Schmidt, J. Kozyra, N. Krupp, M. Fränz, J. Woch, J. Luhmann, S. McKenna-Lawlor, C. Mazelle, J.-J. Thocaven, S. Orsini, R. Cerulli-Irelli, M. Mura, M. Milillo, M. Maggi, E. Roelof, P. Brandt, K. Szego, J.D. Winningham, R.A. Frahm, J. Scherrer, J.R. Sharber, P. Wurz, P. Bochsler, The loss of ions from Venus through the plasma wake. Nature 450, 650-653 (2007). doi:10.1038/nature06434

L.H. Brace, R.F. Theis, W.R. Hoegy, Plasma clouds above the ionopause of Venus and their implications. Planet. Space Sci. 30(1), 29-37 (1982)

J.T. Clarke, G.E. Ballester, J. Trauger, R. Evans, J.E.P. Connerney, K. Stapelfeldt, D. Crisp, P.D. Feldman, C.J. Burrows, S. Casertano, J.S. Gallagher III., R.E. Griffiths, J.J. Hester, J.G. Hoessel, J.A. Holtzman, J.E. Krist, V. Meadows, J.R. Mould, P.A. Scowen, A.M. Watson, J.A. Westphal, Far-ultraviolet imaging of Jupiter's Aurora and the lo "Footprint". Science 274, 404-409 (1996)

S.W.H. Cowley, E.J. Bunce, T.S. Stallard, S. Miller, Jupiter's polar ionospheric flows: Theoretical interpretation. Geophys. Res. Lett. 30(5), 1220 (2003). doi:10.1029/2002GL016030

S. Fukuda, S. Sawai, S. Sakai, H. Saito, T. Nakagawa, T. Tohma, J. Takahashi, K. Kitade, Flexible standard bus for ISAS/JAXA small scientific satellite series, in Proc. of the 26th International Symposium on Space Technology and Science (ISTS) (2008), 2008-f-17

T.W. Hill, Internal limit on corotation. J. Geophys. Res. 84, 6554-6558 (1979)

D.E. Huddleston, C.T. Russell, M.G. Kivelson, K.K. Khurana, L. Bennett, Location and shape of the Jovian magnetopause and bow shock. J. Geophys. Res. 103(E9), 20075 (1998)

N. Iwagami, S. Takagi, S. Ohtsuki, M. Ueno, K. Uemizu, T. Satoh, T. Sakanoi, G.L. Hashimoto, Science requirements and description of the $1 \mu \mathrm{m}$ camera onboard the Akatsuki Venus Orbiter. Earth Planets Space 63, 487 (2011). doi:10.5047/eps.2011.03.007

K.K. Khurana, The influence of solar wind on Jupiter's magnetosphere deduced from currents in the equatorial plane. J. Geophys. Res. 106, 25999 (2001)

N. Krupp, A. Lagg, S. Livi, B. Wilken, J. Woch, E.C. Roelof, D.J. Williams, Global flows of energetic ions in Jupiter's equatorial plane: First-order approximation. J. Geophys. Res. 106, 26017-26032 (2001)

N. Krupp, V.M. Vasyliunas, J. Woch, A. Lagg, K.K. Khurana, M.G. Livelson, B.H. Mauk, E.C. Roelof, D.J. Williams, S.M. Krimigis, W.S. Kurth, L.A. Fransk, W.R. Paterson, Dynamics of the Jovian magnetosphere, in Jupiter, ed. by F. Bagenal, T. Dowling, W. Mckinnon (Cambridge University Press, Cambridge, 2004), p. 617

J.G. Luhmann, J.U. Kozyra, Dayside pickup oxygen ion precipitation at Venus and Mars: Spatial distributions, energy deposition and consequences. J. Geophys. Res. 96, 5457-5467 (1991). doi:10.1029/90JA01753

J.G. Luhmann, A. Fedorov, S. Barabash, E. Carlsson, Y. Futaana, T.L. Zhang, C.T. Russell, J.G. Lyon, S.A. Ledvina, D.A. Brain, Venus express observations of atmospheric oxygen escape during the passage of several coronal mass ejections. J. Geophys. Res. 113, E00B04 (2008). doi:10.1029/2008JE003092

R. Lundin, Ion acceleration and outflow from Mars and Venus: An overview. Space Sci. Rev. 162, 309-334 (2011). doi:10.1007/s11214-011-9811-y

Y. Morita, A year to launch: Japan's epsilon launcher and its evolution, in Proc. of the International Astronautical Congress, vol. 12(D2) (2012), pp. 1-8, x14635

M. Nakamura, T. Imamura, N. Ishii, T. Abe, T. Satoh, M. Suzuki, M. Ueno, A. Yamazaki, N. Iwagami, S. Watanabe, M. Taguchi, T. Fukuhara, Y. Takahashi, M. Yamada, N. Hoshino, S. Ohtsuki, K. Uemizu, G.L. Hashimoto, M. Takagi, Y. Matsuda, K. Ogohara, N. Sato, Y. Kasaba, T. Kouyama, N. Hirata, R. Nakamura, Y. Yamamoto, N. Okada, T. Horinouchi, M. Yamamoto, Y. Hayashi, Overview of Venus orbiter, Akatsuki. Earth Planets Space 63, $443-457$ (2011)

K. Nakaya, S. Fukuda, S. Ssakai, A. Yamazaki, K. Uemizu, T. Toriumi, J. Takahashi, M. Maehara, T. Okahashi, S. Sawai, Development of flexible standard bus for ISAS/JAXA small scientific satellite series, in Proc. of the 29th International Symposium on Space Technology and Science (ISTS) (2011), 2011-f-19

H. Nozawa, H. Misawa, M. Kagitani, F. Tsuchiya, S. Takahashi, A. Morioka, T. Kimura, S. Okano, H. Yamamoto, R. Sood, Implication for the solar wind effect on the Io plasma torus. Geophys. Res. Lett. 33, L16103 (2006). doi:10.1029/2005GL025623

M.J. Reiner, M.L. Kaiser, M.D. Desch, Long-term behavior of Jovian bKOM and nKOM radio emissions observed during the Ulysses-Jupiter encounter. Geophys. Res. Lett. 27, 297-300 (2000)

S. Sakai, A. Yamazaki, K. Uemizu, K. Nakaya, S. Fukuda, S. Sawai, K. Tanaka, Y. Iwayama, Y. Kusakawa, N. Ogura, Utilization of a mission sensor in the attitude control loop: A cost effective approach for high pointing accuracy, in Proc. of the 29th International Symposium on Space Technology and Science (ISTS) (2011), 2011-f-20 
S. Sawai et al., Overview of a first small scientific satellite of HISAKI (SPRINT-A). Earth Planets Space (2014, in preparation)

A.J. Steffl, A.I.F. Stewart, F. Bagenal, Cassini UVIS observations of the Io plasma torus. I. Initial results. Icarus 172, 78-90 (2004)

T. Tanaka, K. Murawski, Three-dimensional MHD simulation of the solar wind interaction with the ionosphere of Venus: Results of two-component reacting plasma simulation. J. Geophys. Res. 102, 1980519822 (1997). doi:10.1029/97JA01474

C. Tao, R. Kataoka, H. Fukunishi, Y. Takahashi, T. Yokoyama, Magnetic field variations in the Jovian magnetotail induced by solar wind dynamic pressure enhancements. J. Geophys. Res. 110, A11208 (2005). doi:10.1029/2004JA010959

N. Terada, S. Machida, H. Shinagawa, Global hybrid simulation of the Kelvin-Helmholtz instability at the Venus ionopause. J. Geophys. Res. 107, SMP 30-1 (2002). doi:10.1029/2001JA009224

N. Terada, Y.N. Kulikov, H. Lammer, H.I.M. Lichtenegger, T. Tanaka, H. Shinagawa, T. Zhang, Atmosphere and water loss from early Mars under extreme solar wind and EUV conditions. Astrobiology 9, 55 (2009a). doi:10.1089/ast.2008.0250

N. Terada, H. Shinagawa, T. Tanaka, K. Murawski, K. Terada, A three-dimensional, multispecies, comprehensive MHD model of the solar wind interaction with the planet Venus. J. Geophys. Res. 114, A09208 (2009b). doi:10.1029/2008JA013937

N. Thomas, F. Bagenal, T.W. Hill, J.K. Wilson, The Io neutral clouds and plasma torus, in Jupiter, ed. by F. Bagenal, T. Dowling, W. Mckinnon (Cambridge University Press, Cambridge, 2004), p. 561

F. Tsuchiya, M. Kagitani, N. Terada, Y. Kasaba, I. Yoshikawa, G. Murakami, K. Sakai, T. Homma, K. Yoshioka, A. Yamazaki, K. Uemizu, T. Kimura, M. Ueno, Plan for observing magnetospheres of outer planets by using the EUV spectrograph onboard the SPRINT-A/EXCEED mission. Adv. Geosci. 25, 57 (2011)

M. Ueno, PtSi infrared camera for astronomical observations. SPIE J. 2744, 84-91 (1996)

I. Yoshikawa, K. Yoshioka, G. Murakami, A. Yamazaki, F. Tsuchiya, M. Kagitani, T. Sakanoi, T. Kimura, M. Kuwabara, K. Fujiwara, T. Hamaguchi, H. Tadokoro, Extreme ultraviolet radiation measurement for planetary atmospheres/magnetospheres from the Earth-orbiting spacecraft (Extreme Ultraviolet Spectroscope for Exospheric Dynamics: EXCEED). Space Sci. Rev. (2014, submitted). doi:10.1007/s11214-014-0077-z

K. Yoshioka, G. Murakami, A. Yamazaki, F. Tsuchiya, M. Kagitani, T. Sakanoi, T. Kimura, K. Uemizu, K. Uji, I. Yoshikawa, The extreme ultraviolet spectroscope for planetary science, EXCEED. Planet. Space Sci. 85, 250-260 (2013) 